\title{
GT2015-43389
}

\section{EFFECTS OF UNSTEADY FLOW INTERACTIONS ON THE PERFORMANCE OF A HIGHLY-LOADED TRANSONIC COMPRESSOR STAGE}

\author{
Chunill Hah
}

NASA Glenn Research Center,

MS 5-10, Cleveland, Ohio 


\section{Background}

- NASA ERA Program

- Physics of Loss Generation in a GE

Highly Loaded Transonic Compressor.

- Aero Testing at NASA/Glenn W7 facility.

- NASA Internal CFD study with RANS, URANS, LES. 


\section{CFD analysis of the first stage}

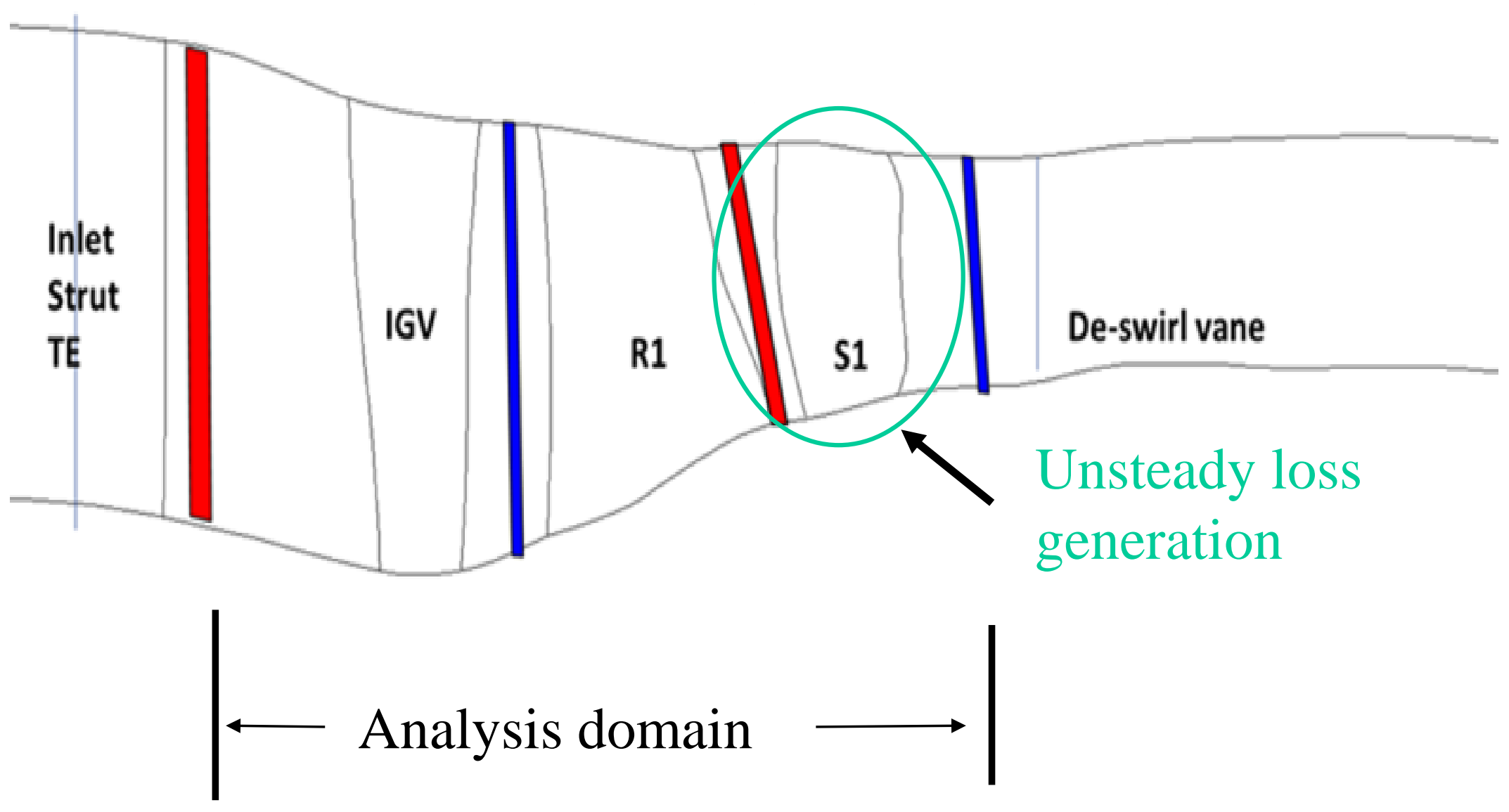




\section{Objectives}

- Can a high-fidelity CFD (LES) calculate measured loss generation in Stator 1 ?

- Understand flow mechanism for this unsteady loss generation due to the incoming rotor wake.

- Possible ways to reduce loss generation? 


\section{Order of presentation}

- LES set-up and CFD grids.

- Compressor characteristics from LES.

- Unsteady loss generation in the stator passage.

- Concluding remarks. 


\section{LES for turbomachinery application}

- To address some shortcomings of RANS/URANS (vortex interaction, flow separation, wake development. Etc.)

- Significant increase in computing cost with large size computational grid.

- Solution depends on CFD grid.

- Good insight and knowledge required to extract physics (needs further development). 


\section{Applied LES procedure}

- $3^{\text {rd }}$-order scheme for convection terms.

- $2^{\text {nd }}$-order central differencing for diffusion terms.

- Sub-iteration at each time step.

- Dynamic model for sub grid stress tensor. 


\section{LES Set-Up}

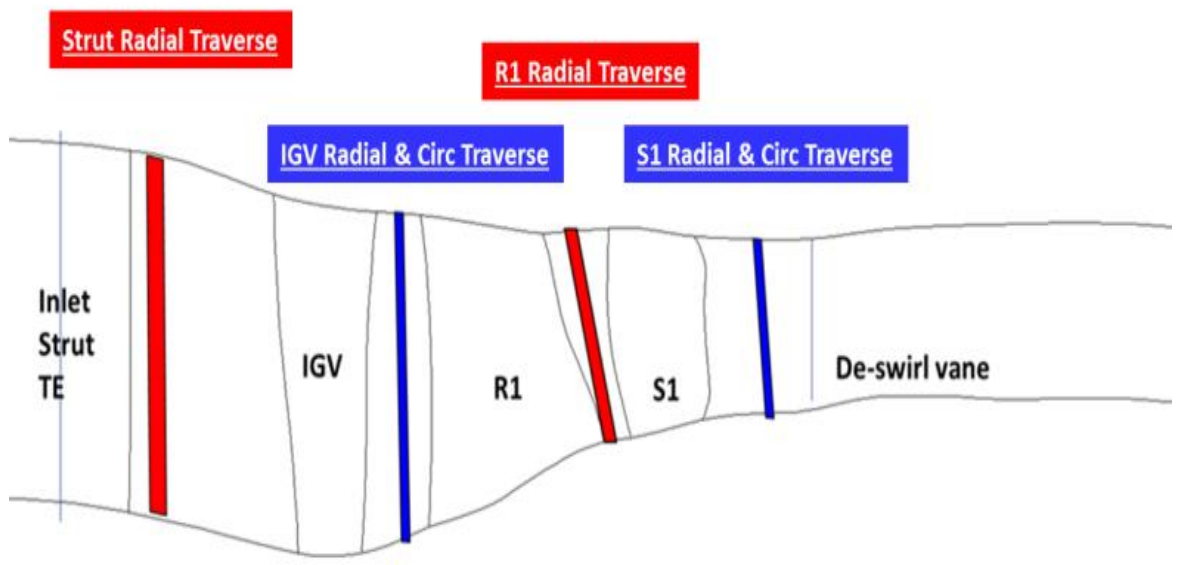

- Original Blades : 42 IGV, 28 R1, and 58 S1.

Scaled to 42 IGV, 28 R1, and 56S1.

- 3 IGV , 2 R1 , and 4 S1 passages analyzed with periodicity condition.

- 500 million CFD nodes for 9 passages (for S1, 384x356x650 in B to B, Spanwise, axial direction for each passage) 


\section{Computational grid and domain}
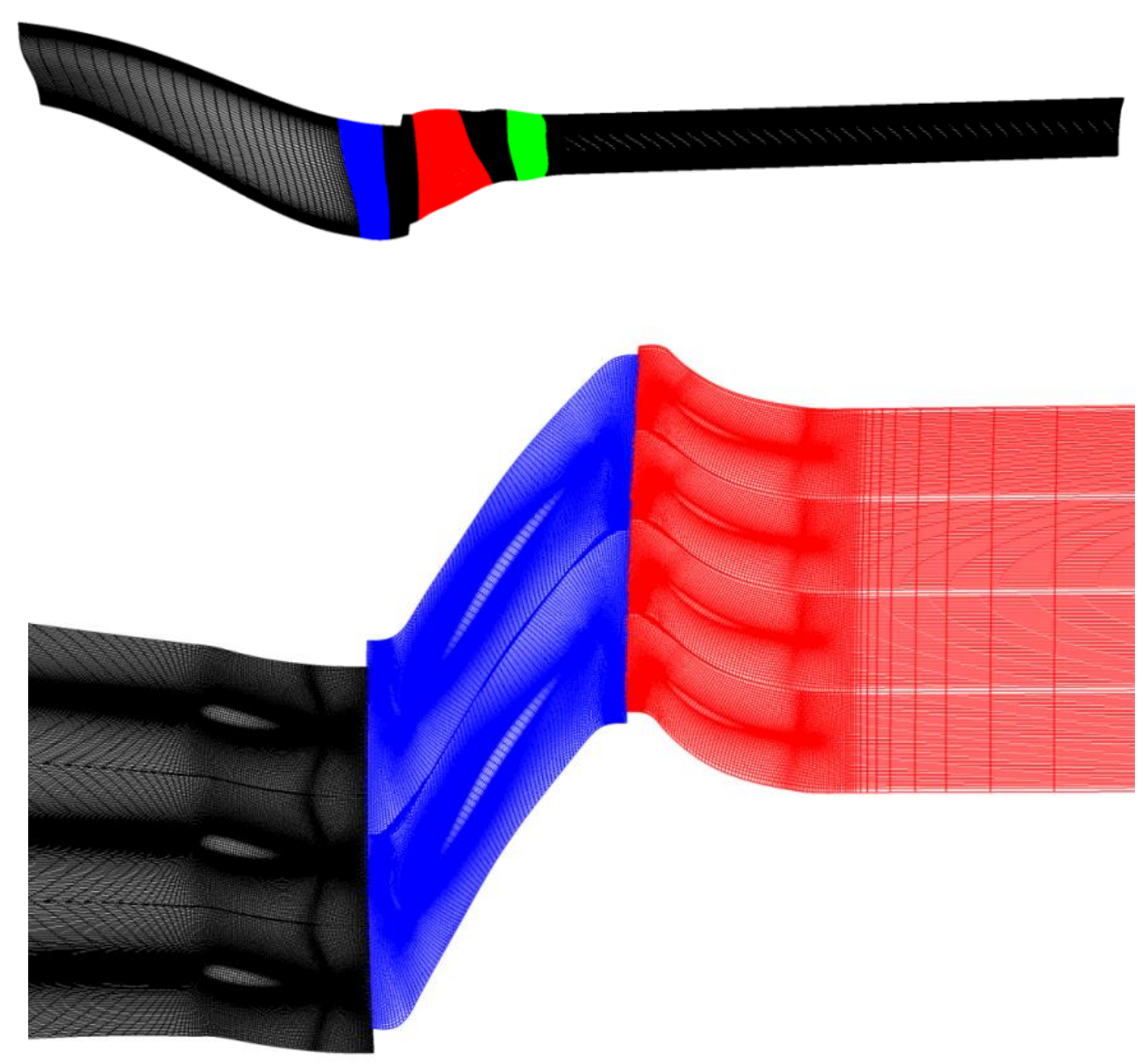

IGV

Rotor 1

Stator 1 


\section{Overall compressor flow field from LES}




\section{Instantaneous pressure distribution at mid-span}

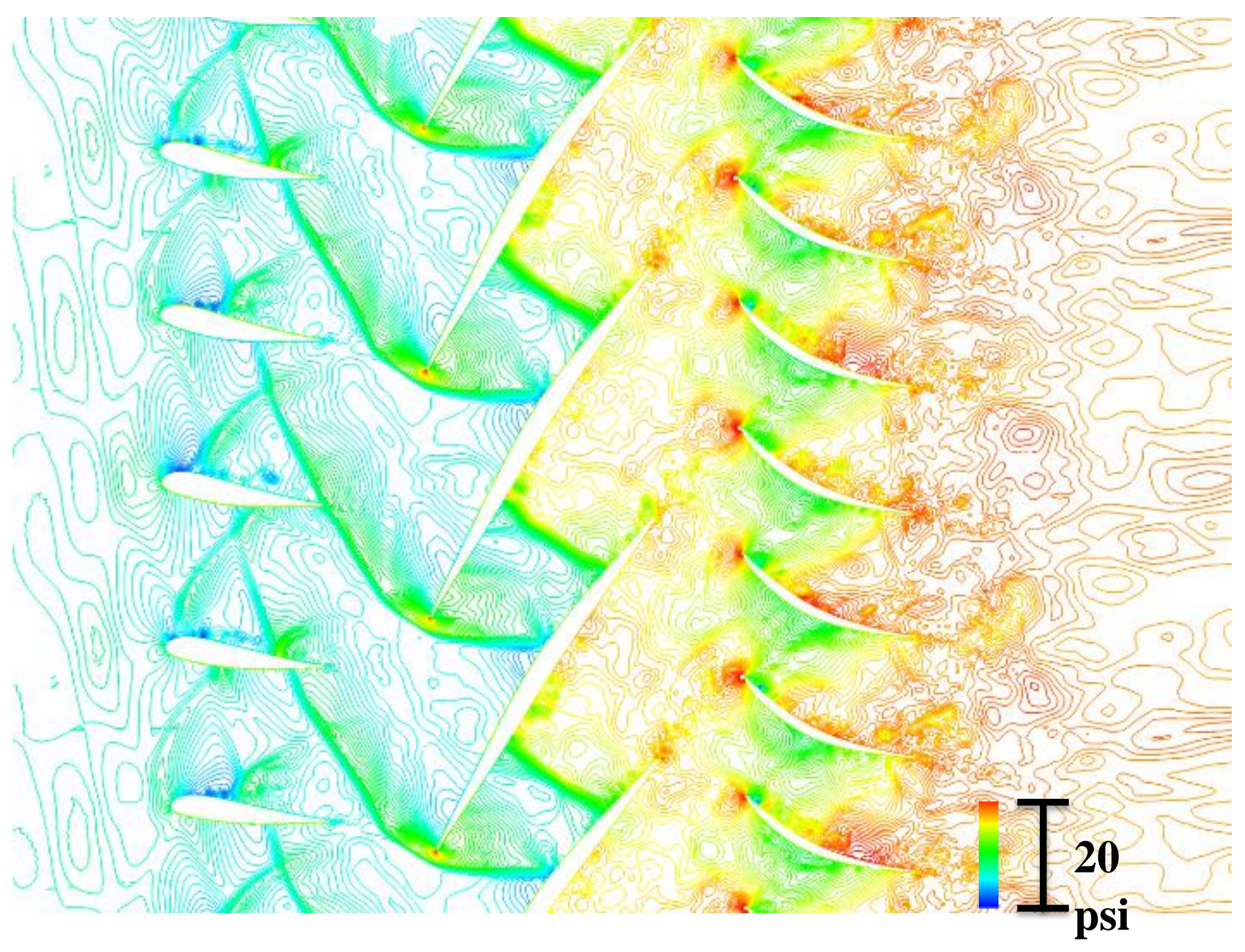




\section{Instantaneous vorticity distribution at mid-span}

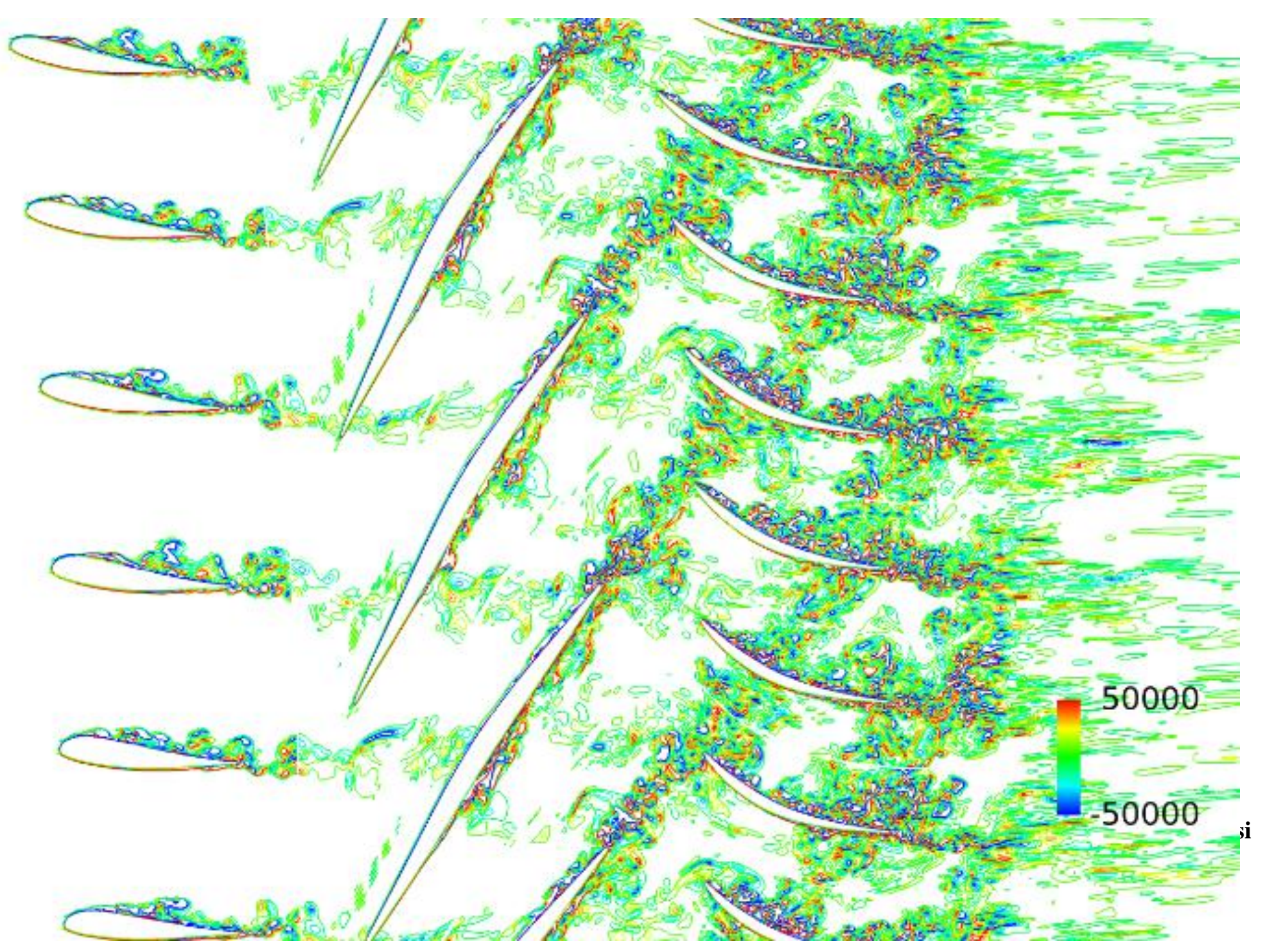




\section{Comparison of corrected speedline relative}

to multi-stage compressor opline
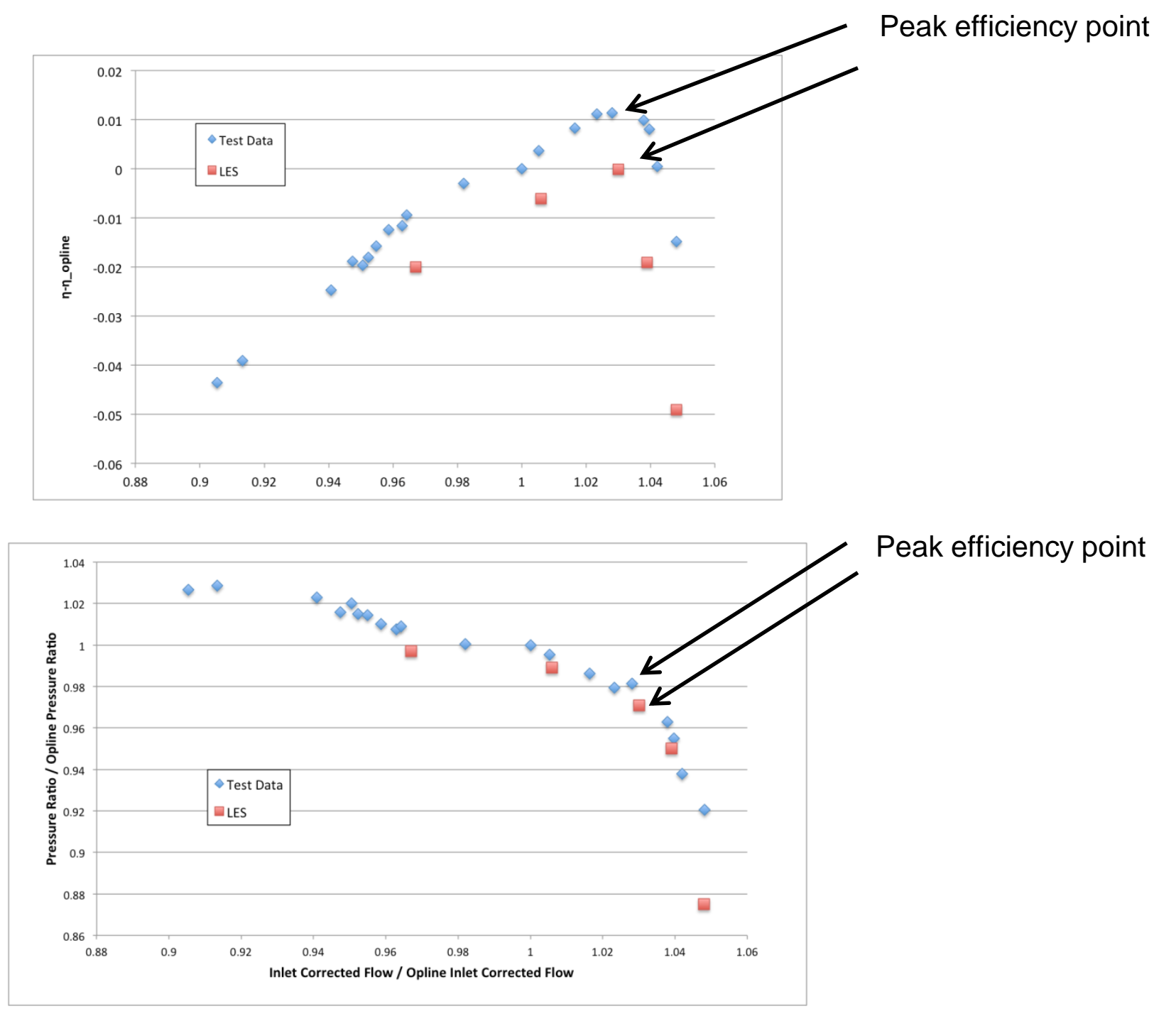


\section{Comparison of IGV exit swirl angle}

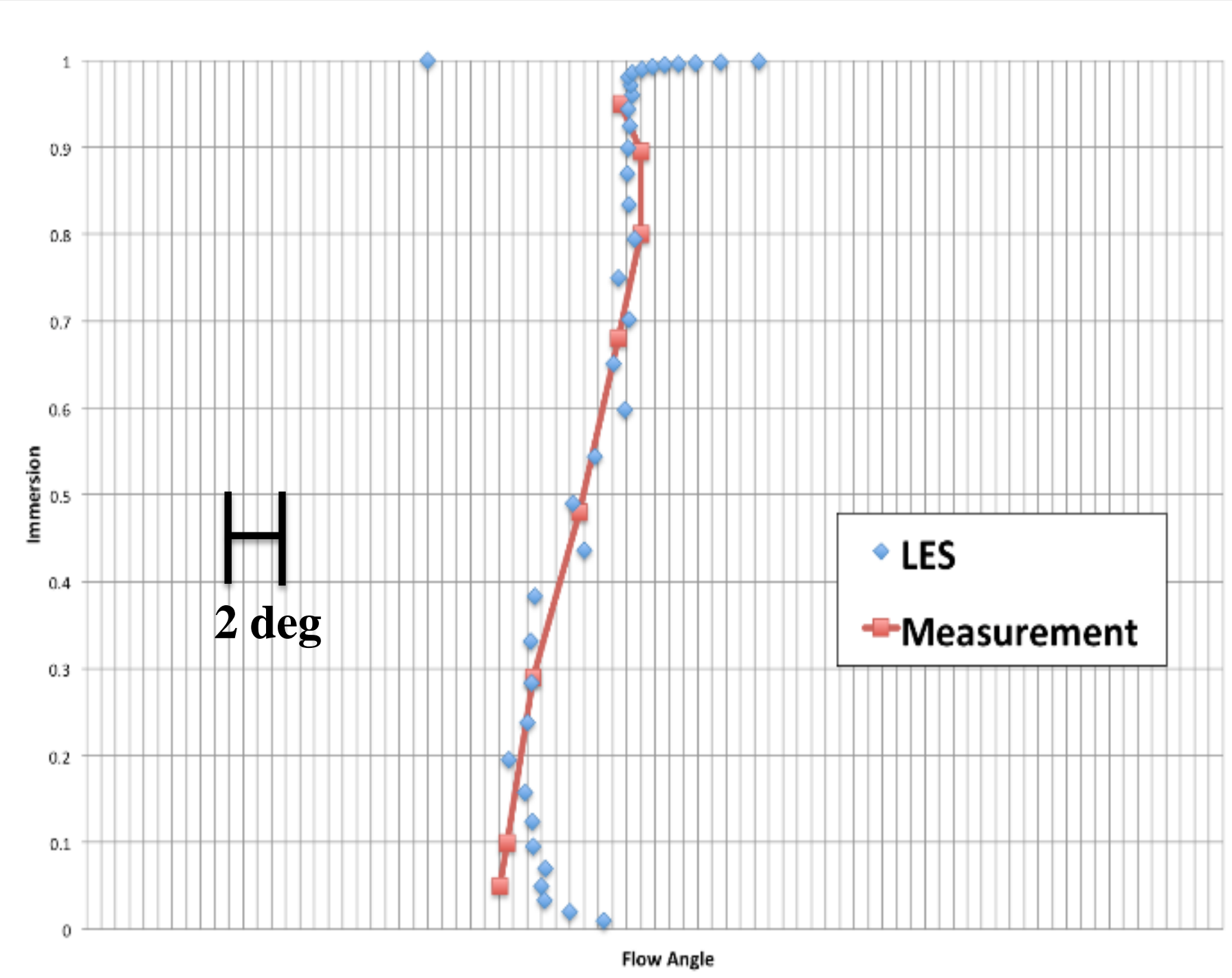




\section{Comparison of total pressure at IGV exit}

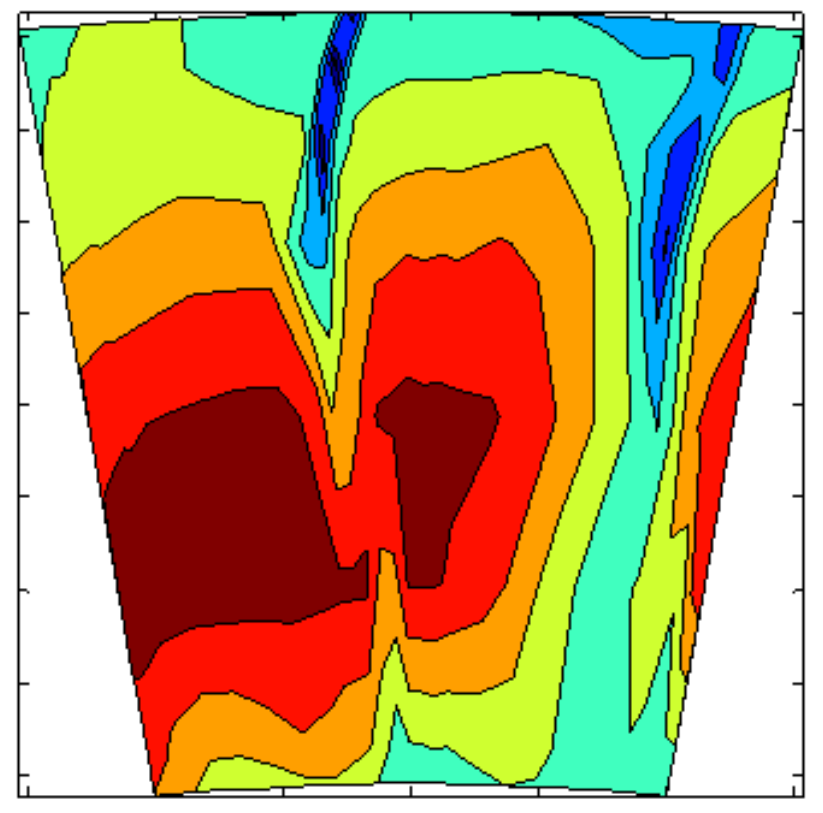

Contours at $0.2 p s i$ Increments

5-hole traverse

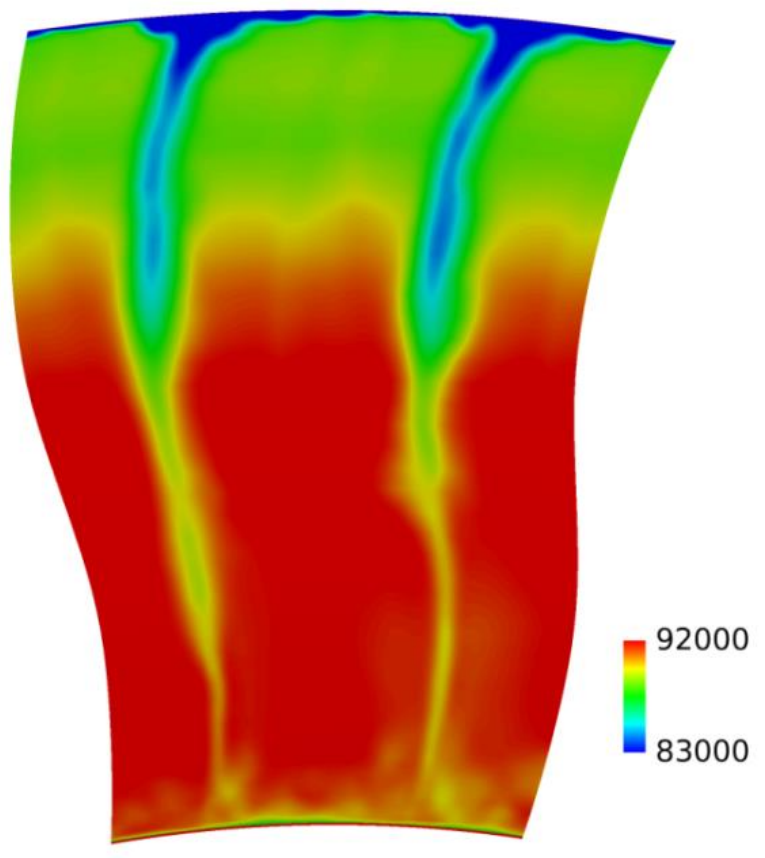

LES 


\section{Comparison of Pt and Tt at exit of R1 and S1}
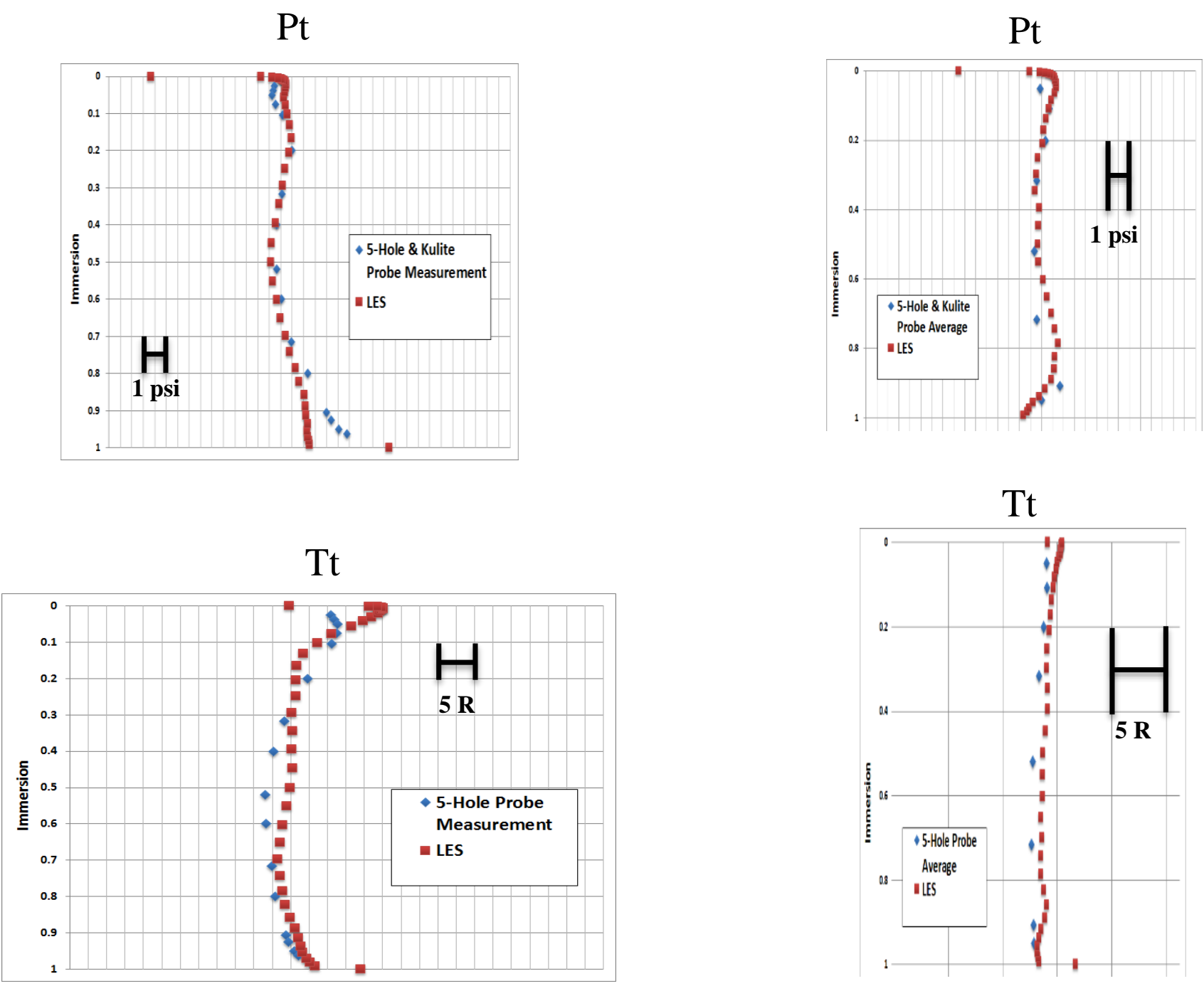

R1 exit

S1 exit 


\section{Unsteady loss generation in the stator due to incoming rotor wake}




\section{Measured Pt and Tt at stator exit}
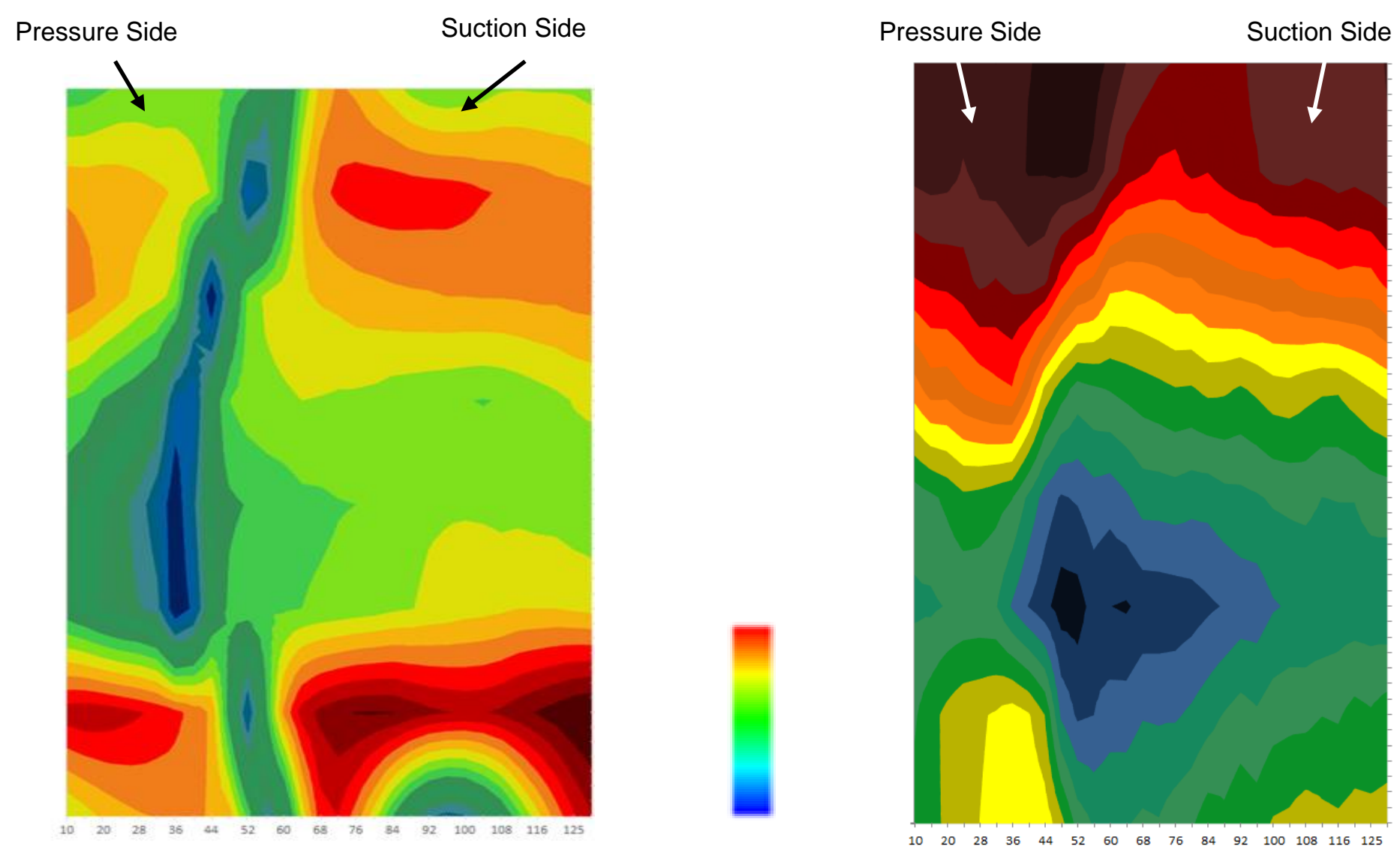

$\mathrm{Pt}$

$\mathrm{Tt}$ 
Measured Pt, Tt, and entropy at $48.1 \%$ span (Lurie and Breeze-Stringfellow[GT2015-42526])

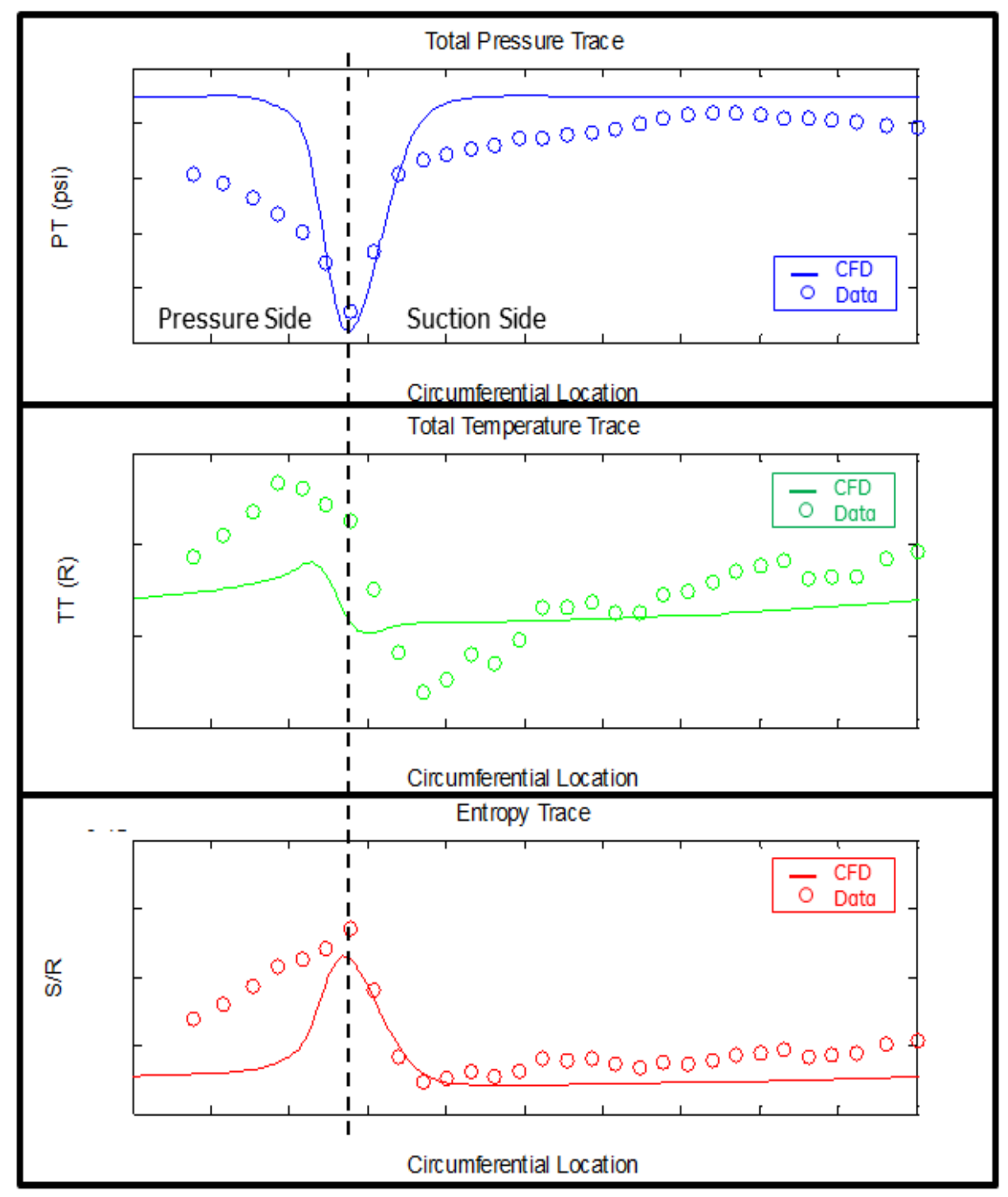




\section{Comparison of Pt from LES, S1 exit}
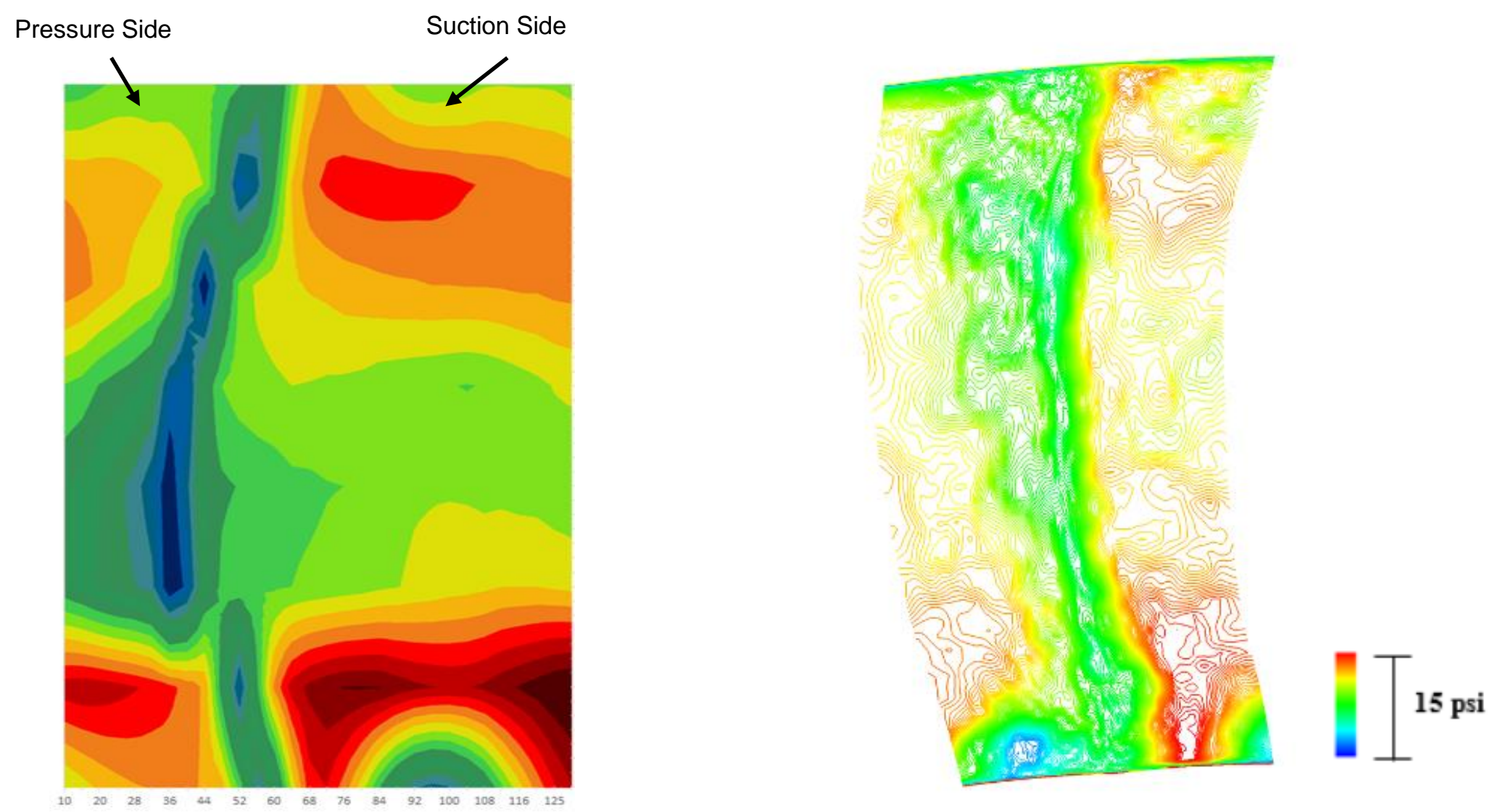

Five hole probe

LES 


\section{Comparison of $\mathrm{Tt}$ from LES, S1 exit}
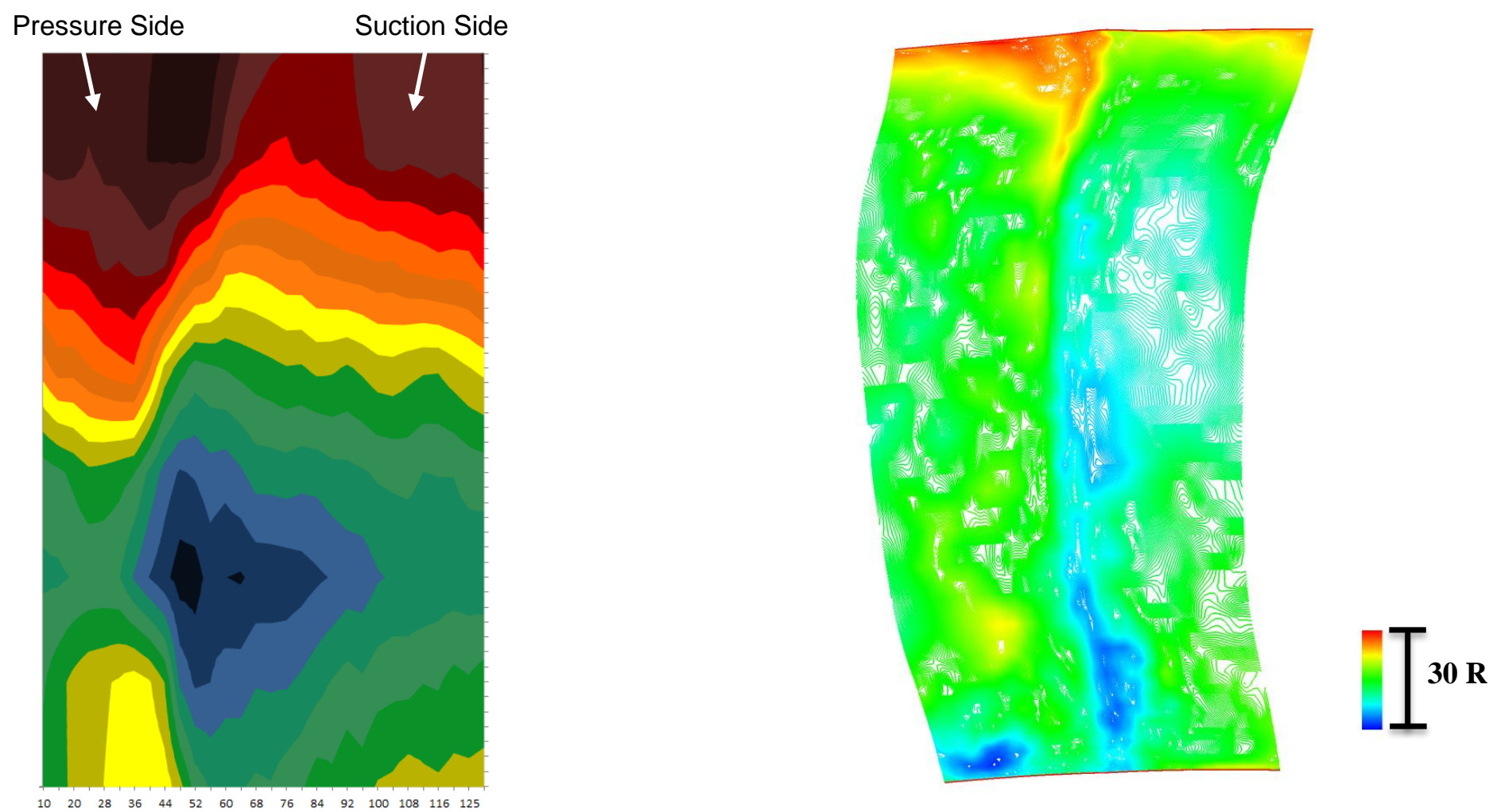

Measurement

LES 


\section{Comparison of Pt and $\mathrm{Tt}$ at mid-span}
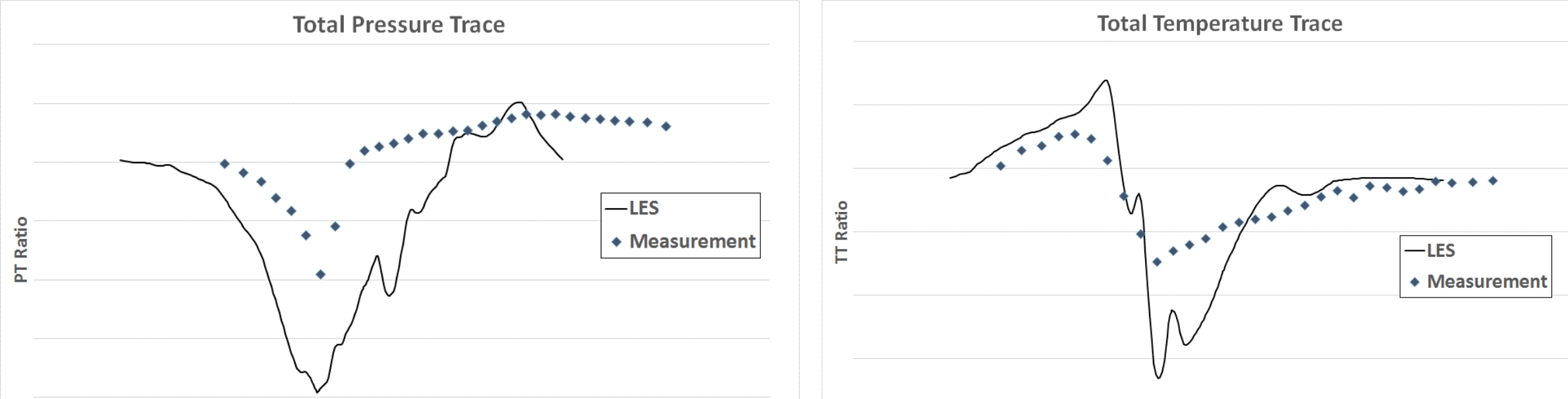

Circumferential Location

Circumferential Location 


\section{Instantaneous distribution of Pt from LES}

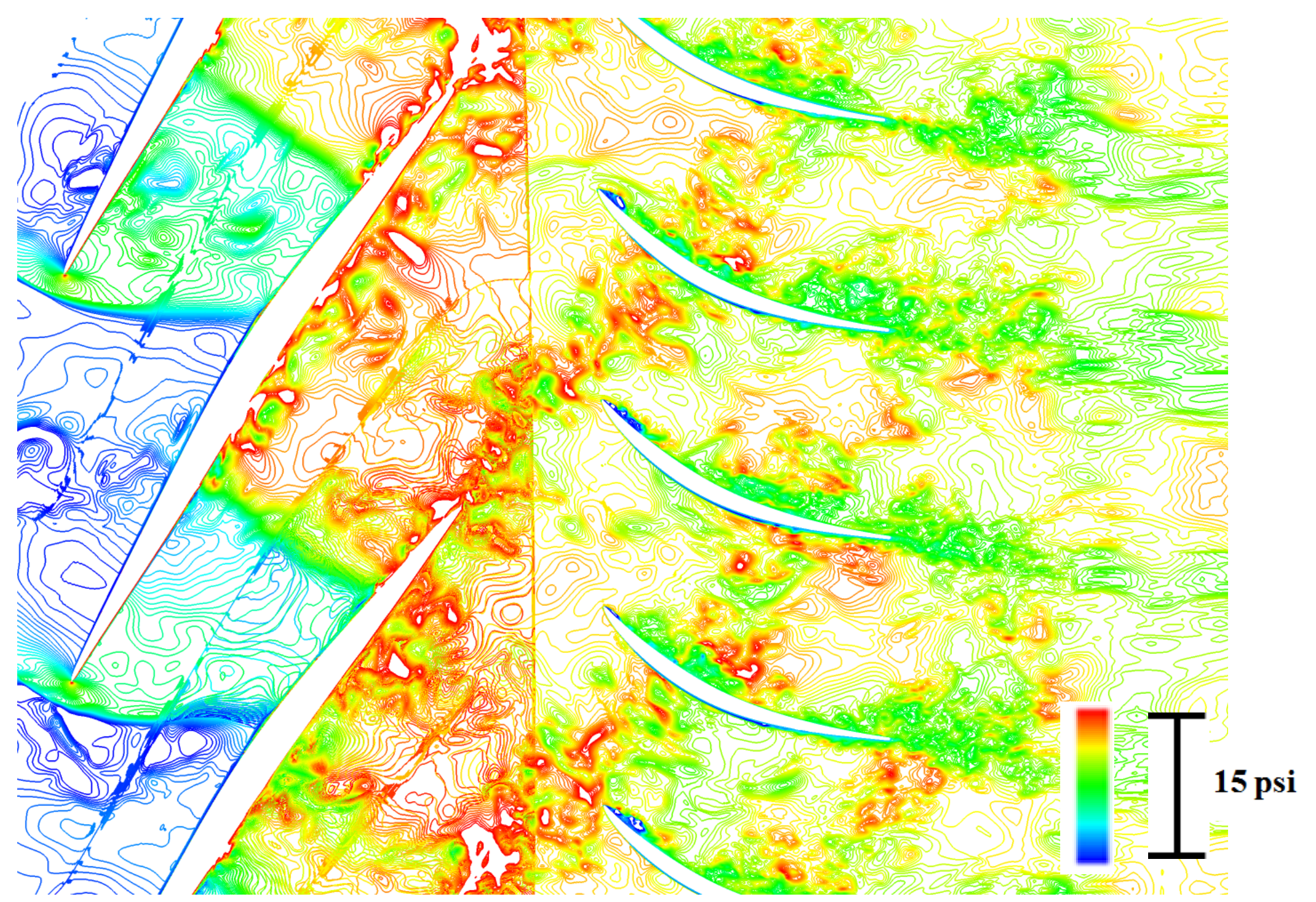




\section{Pt time-space plot at S1 exit}

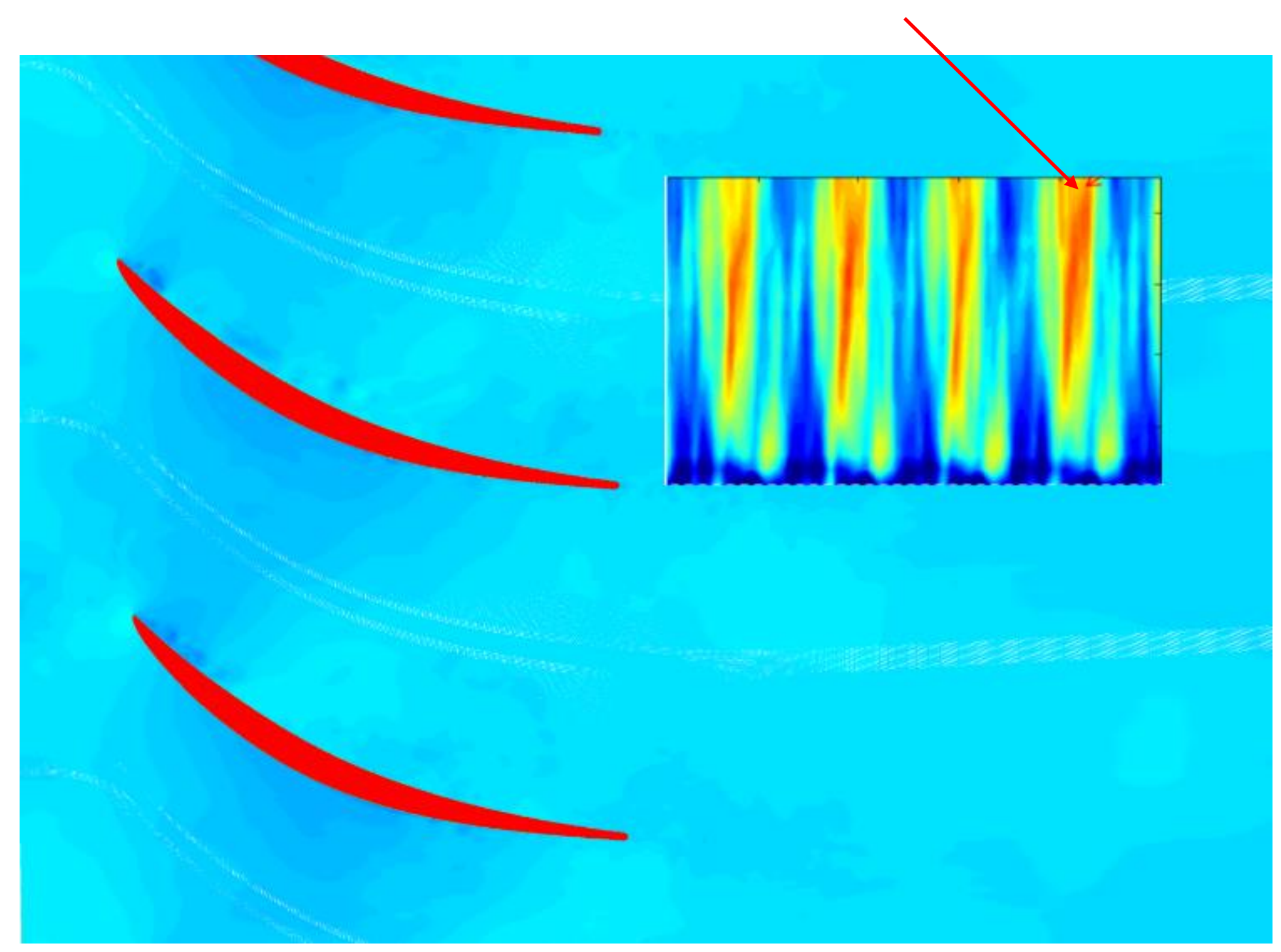




\section{Instantaneous distribution of Tt from LES}

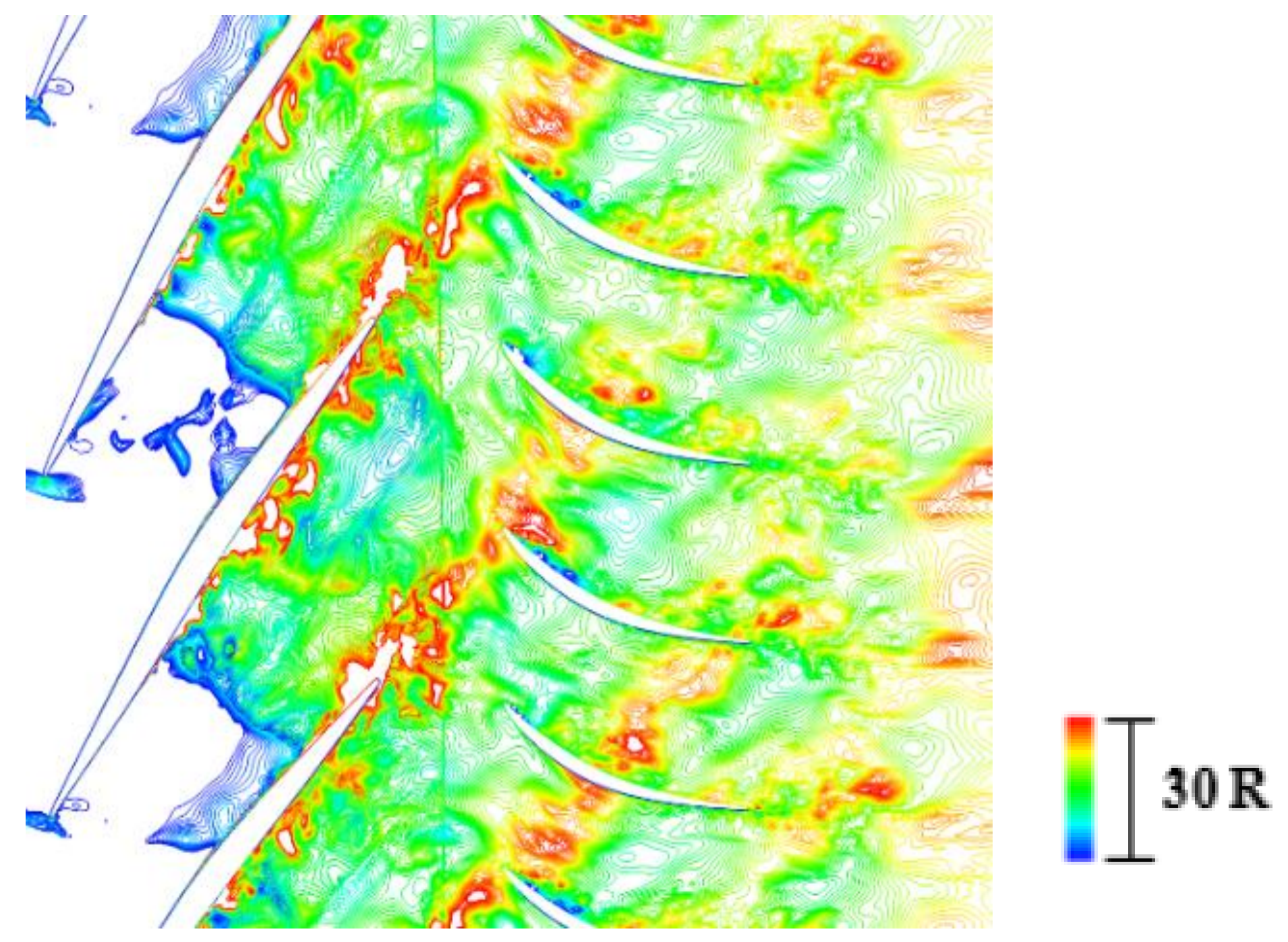




\section{Why higher Tt and lower Pt on the pressure side of the stator?}

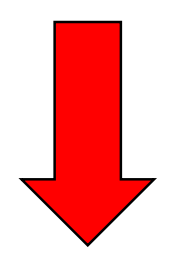

Why URANS does not pick up this trend?

Why LES shows the correct trend ?

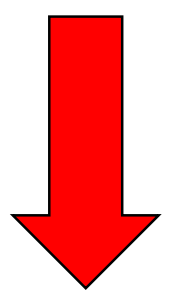

Flow mechanism for unsteady loss generation 


\section{Loss generation in multi-stage compressors}

Smith, L.H. Jr. : Wake Dispersion, 1966.

Kerrebrock, J.L. and Mikolajczk, A.A. :

Intra-Stator transport of rotor wakes, 1970
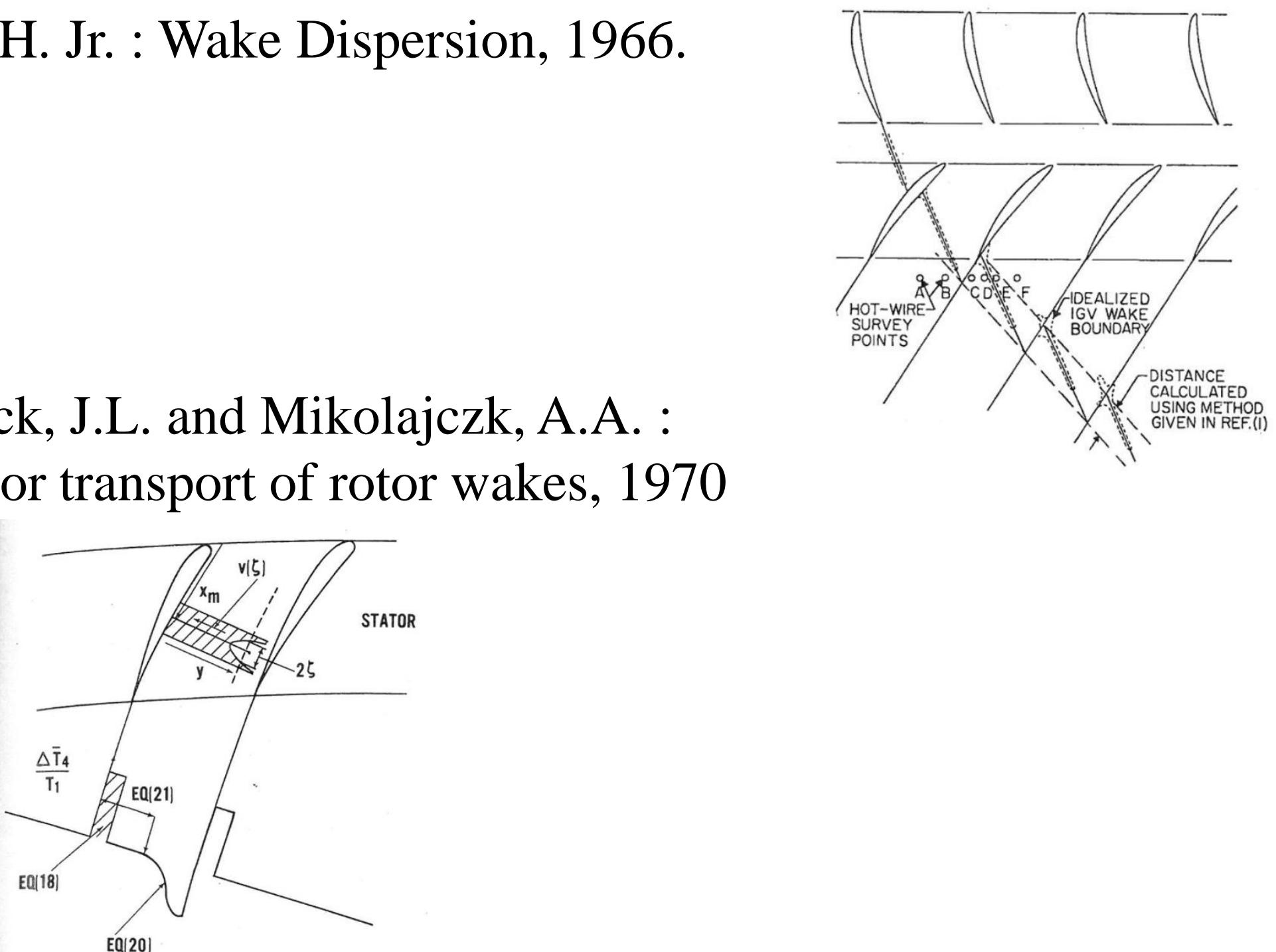


\section{Instantaneous velocity vectors at mid-span}
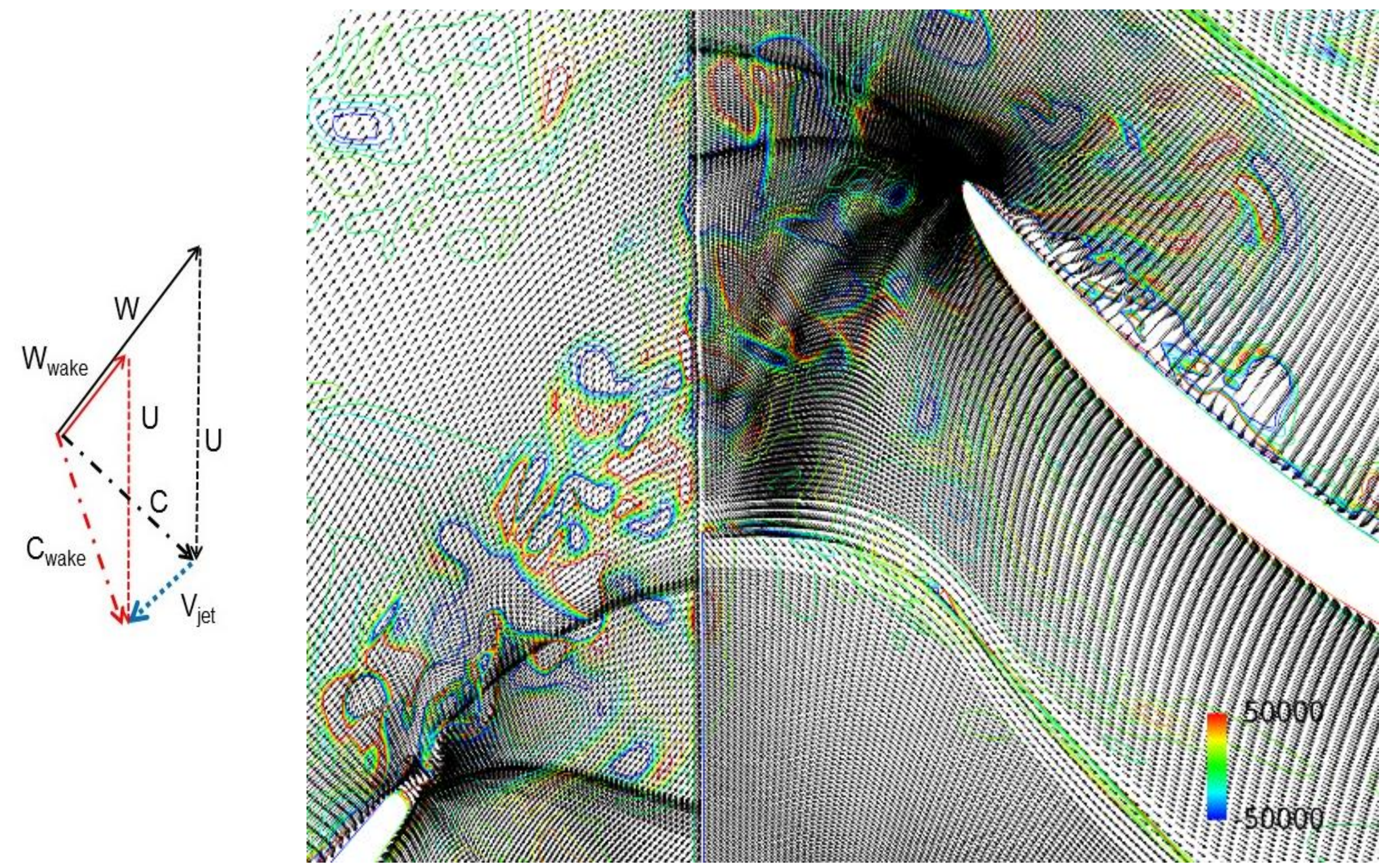


\section{Velocity vectors in rotor wake}

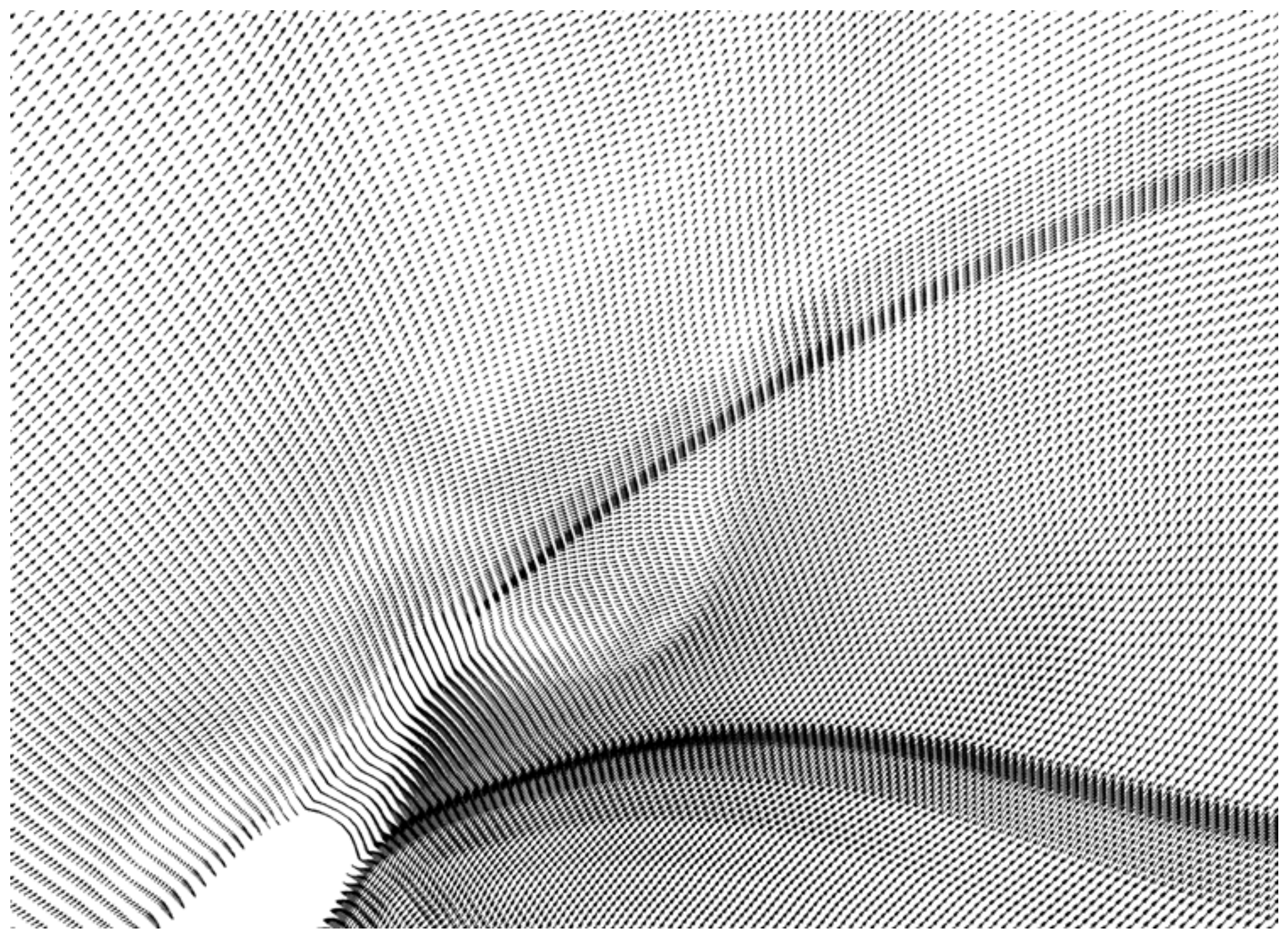




\section{Absolute Pt in the rotor wake}

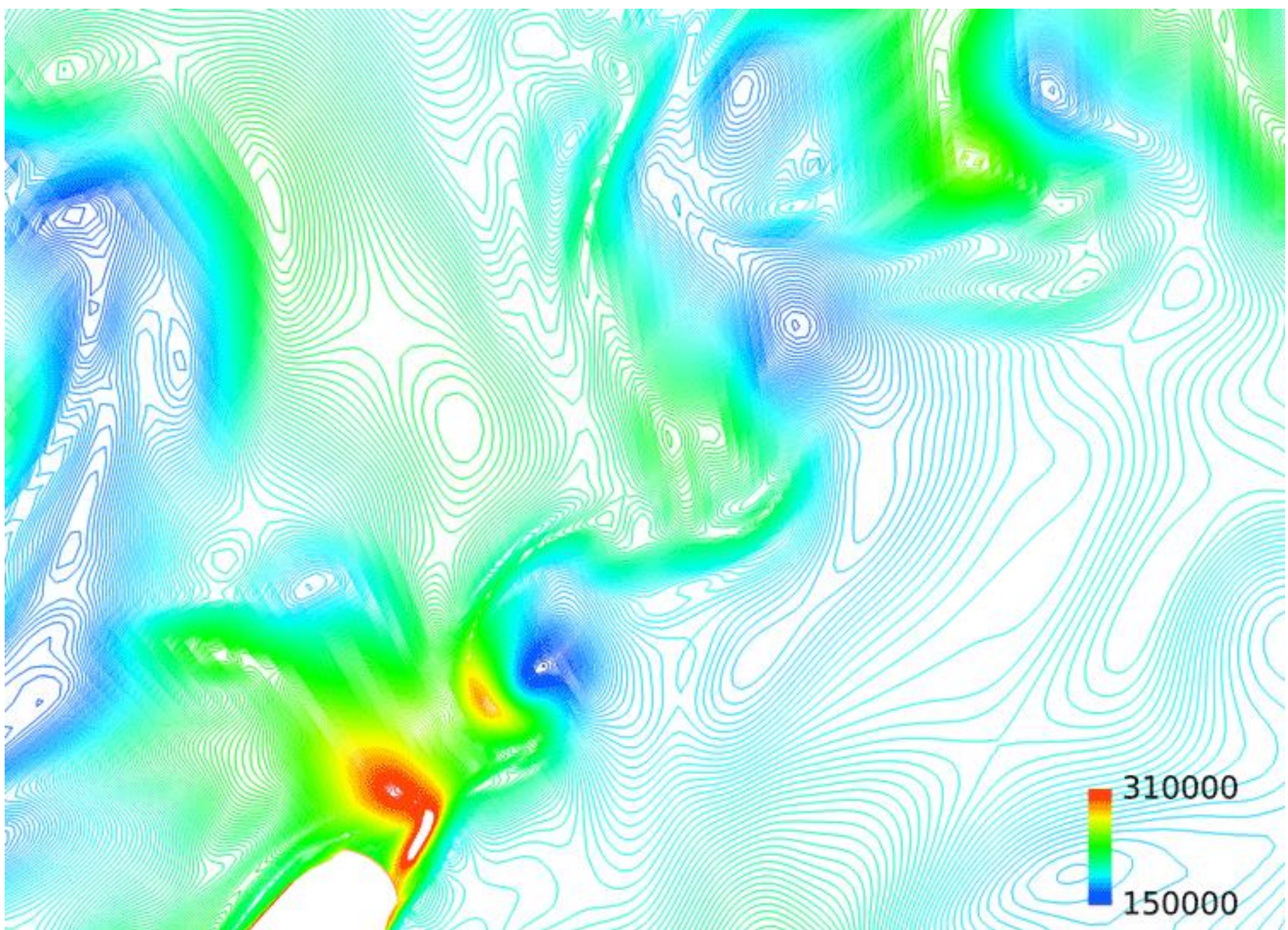




\section{Instantaneous tangential velocity component in stator frame}

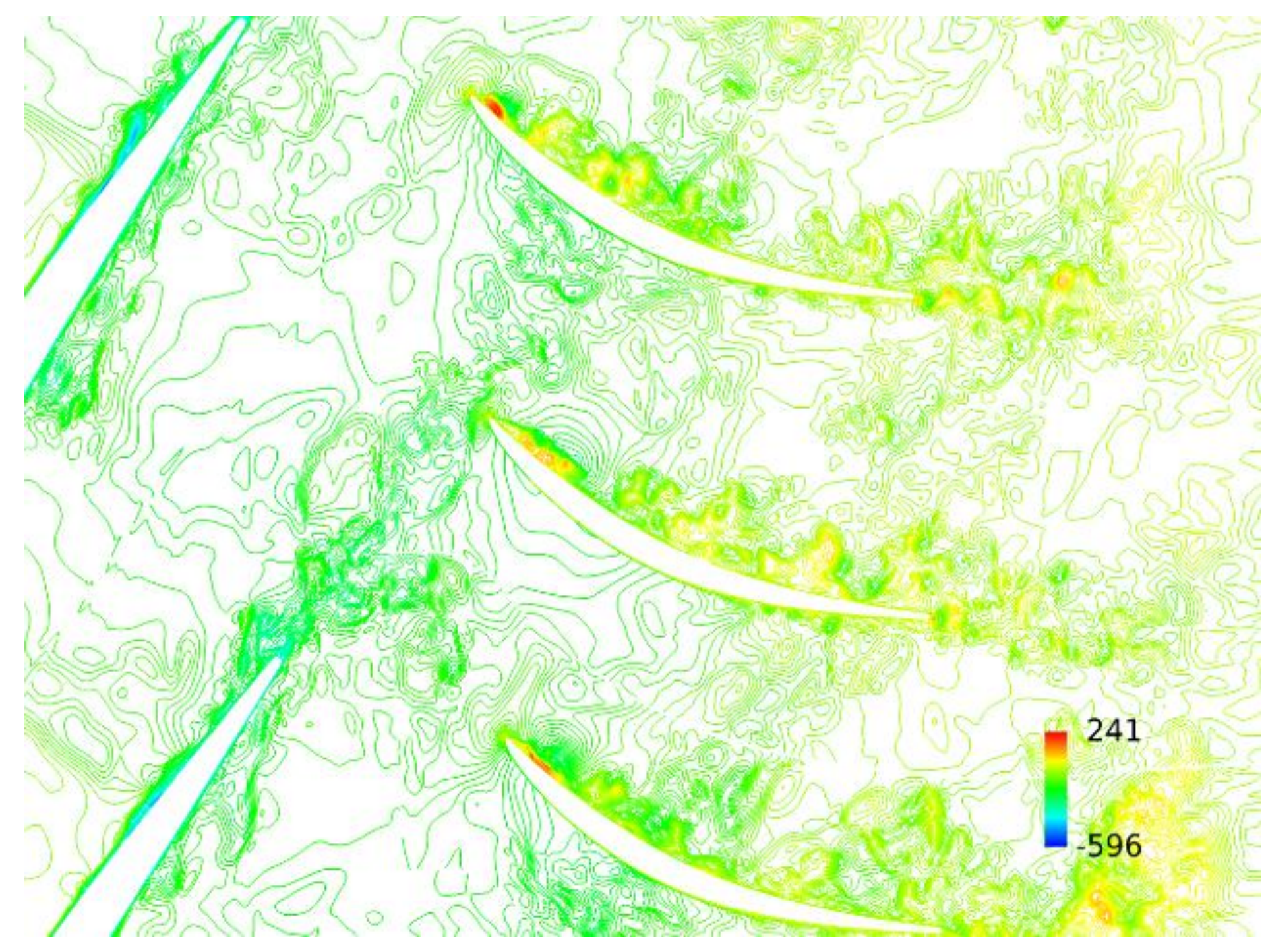




\section{Intra-stator transport of rotor wake for high Tt on PS}

Both $\mathrm{Tt}$ and $\mathrm{Pt}$ are higher in rotor wake for the current compressor.

Jet velocity in the rotor wake decays very fast and The rotor wake is not like 2-D inviscid wake.

What makes Tt higher on pressure side of S1 ?

Why $\mathrm{Pt}$ is lower on pressure side of $\mathrm{S} 1$ ? 


\section{Changes of Pt inside the S1}

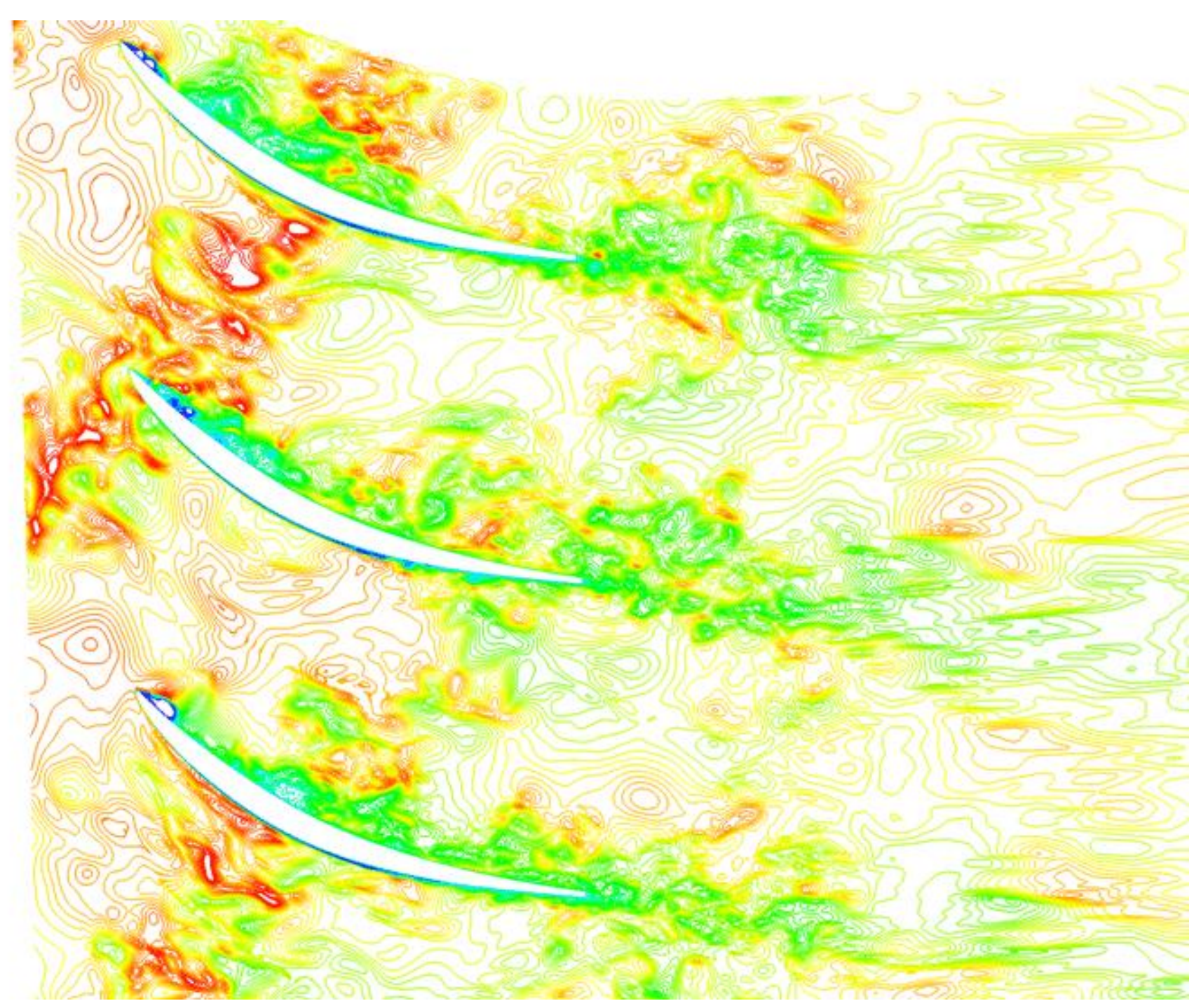




\section{Changes of Tt inside the S1}

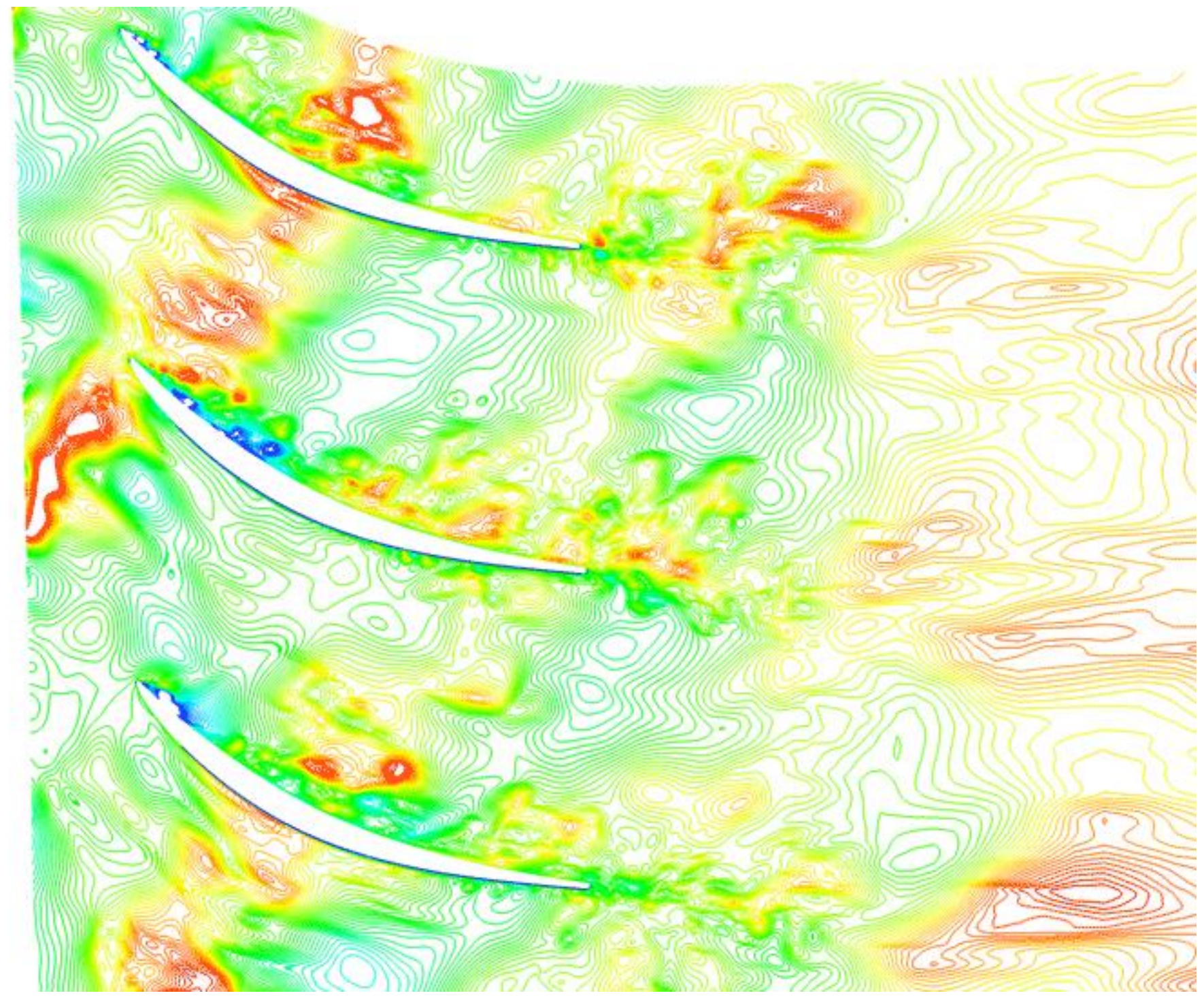


Changes of Vorticity inside the S1

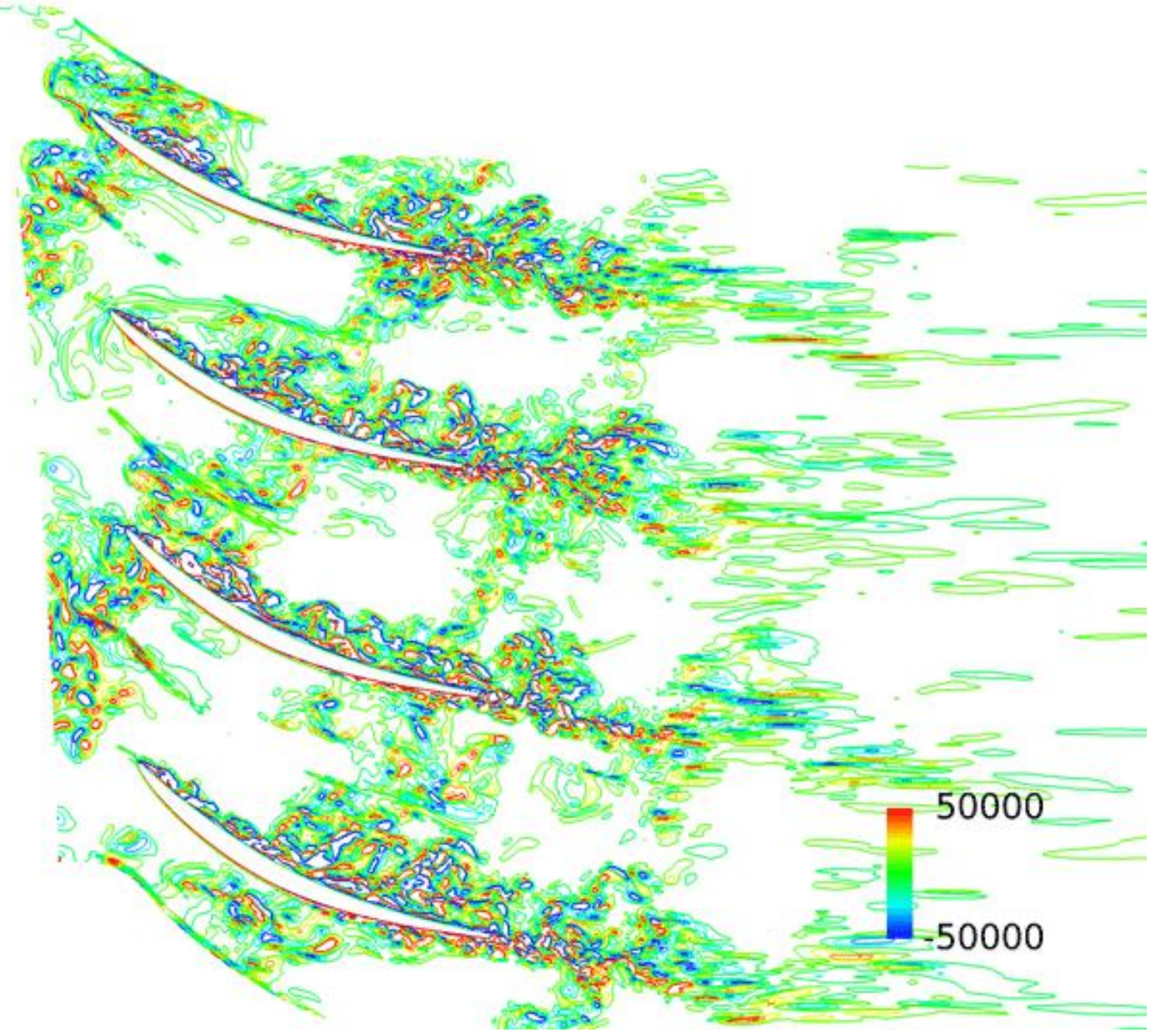




\section{Possible mechanism of high $\mathrm{Tt}$}

\section{and low Pt (higher loss) on}

\section{pressure side of S1}

- Higher measured Tt at S1 exit might not be due to inviscid intra-stator transport of rotor wake for this compressor. (jet velocity decays fast, URANS does not calculate Higher Tt on PS).

- Different 3-D unsteady vortex interactions near SS and PS due to curvature effects.

- Wake stretching contributes wider rotor wake near the PS (higher Tt at S1 exit). 


\section{Mechanisms of unsteady loss generation}
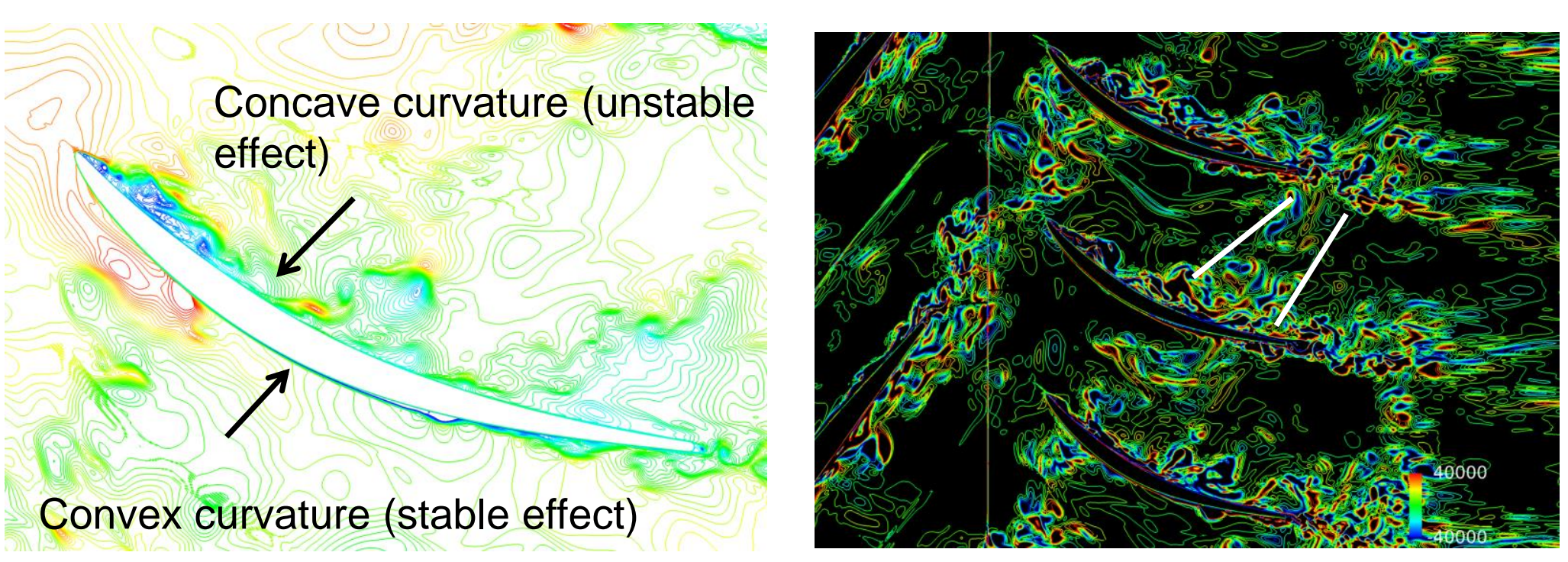

Curvature effects

Wake stretching 


\section{Concluding remarks}

- Investigated unsteady loss generation in the stator passage due to incoming rotor wake.

- Three-dimensional unsteady vortex interaction seems to be the main reason for the high loss near the pressure side of the stator.

- Further focused experimental/analytical studies will lead to advanced designs. 